WE3B-3

\title{
Design of an LTCC Tri-Band Transceiver Module for GPRS Mobile Applications
}

\author{
Yo-Shen Lin, Chien-Chang Liu*, Ko-Mai Li*, and Chun Hsiung Chen \\ Department of Electrical Engineering and Graduate Institute of Communication Engineering, \\ National Taiwan University, Taipei 106, Taiwan \\ *Hardware Design Department, Chi-Mei Communication System Inc., Taipei 106, Taiwan
}

\begin{abstract}
This paper presents the results of a lowtemperature co-fired ceramic (LTCC) module for tri-band GPRS mobile applications. The proposed transceiver module integrates the tri-band (EGSM/DCS/PCS) antenna switch, surface acoustic wave (SAW) filters for band selection, and a fully integrated tri-band transceiver IC into one LTCC package. Integrated passive devices include the diplexer, lowpass filters for harmonic rejection, transmission lines, and circult elements for wiring/impedance matching. The module size is $13.8 \mathrm{~mm} \times 8.1 \mathrm{~mm}$, with a packaged height of $1.8 \mathrm{~mm}$. The module is compliant with the ETSI regulations and is currently working on commercially available mobile phones.

Index Terms - Ceramic, LTCC, transceiver, module, GSM, GPRS.
\end{abstract}

\section{INTRODUCTION}

Driven by the demand for small-size and lightweight mobile communication devices, there is an increasing interest in developing miniature RF and microwave frontend modules. Recent advances in integration technology and device performance have paved the way for higher level of system integration on chip (SOC) or on package (SOP). A complete radio front-end for mobile communication devices includes several blocks, such as the transceiver IC, power amplifier, antenna front-end module, control circuitry, and impedance matching components. The electrical specifications for each block dictate the technology to achieve the stringent system performance requirements. In the present state-of-the-art mobile communication systems, Si CMOS/BiCMOS and GaAs HBT are used for transceiver and power amplifier IC implementation, respectively; while ceramic and laminate substrates are used for passive components and circuits, in addition to the SAW technology necessary for filtering. The application of advanced packaging technology such as LTCC enables the integration of these blocks into one single package such that smaller size and lightweight can be achieved [1]-[4]. In [4], the LTCC substrate has been successfully implemented to integrate the tri-band receiver front-end for global system for mobile communications (GSM) applications into one single LTCC package.

In this work, we take a step further on the integration level, and implement a very compact LTCC RF transceiver module for tri-band (EGSM/DCS/PCS) general packet radio service (GPRS) mobile applications. The vertical integration capability of LTCC substrate and the adoption of transceiver IC in bare die form lead to the compact module size of $13.8 * 8.1 * 1.8 \mathrm{~mm}^{3}$.

\section{Transceiver Module Block Diagram}

Shown in Fig. 1 is the block diagram of the proposed tri-band transceiver module. It integrates almost all the radio front-end components of a tri-band GPRS mobile phone, except for the power amplifier module, into one single LTCC package. It is composed of an antenna switch, a set of three SAW filters for each frequency band, and a direct conversion receiver-based RF transceiver IC. The receive path of the proposed transceiver module consists of a tri-band antenna switch, three band-selection SAW filters, and the receive section of the RF transceiver IC. The receive section of the transceiver IC contains for each band an integrated LNA, a quadrature demodulator section performing direct down conversion, baseband amplifier/filter circuitry with I and Q outputs, and three stages of DC offset correction. On the transmit path of the transceiver module, it is comprised of the same tri-band antenna switch as in the receive path, with two embedded low-pass filters for harmonic suppression, and the transmit section of the RF transceiver IC. For the transmit section of transceiver IC, it consists of a vector modulator within a translation loop (offset PLL) architecture with integrated high-power transmit oscillators for frequency upconversion. The synthesizer is also integrated in the transceiver IC and is driven by the external crystal oscillator. Band selection of the proposed transceiver module is performed externally through the bi-directional input. To control different modes of operation, a 24-bit 
word register is programmed using the three-wire input signals. By utilizing the vertical integration capability of LTCC, the proposed transceiver module is realized in one single LTCC package. With an external tri-band power amplifier module, reference oscillator, and voltage regulators, the proposed transceiver module will then form the complete RF circuitry for a tri-band GPRS mobile phone.

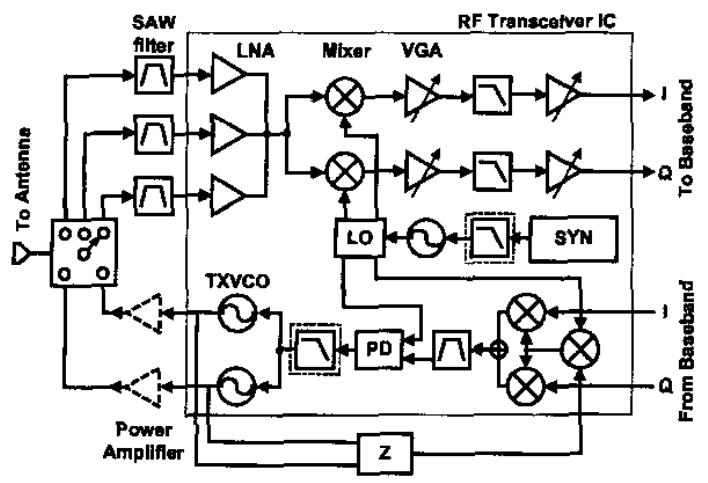

Fig. 1. Block diagram of the proposed tri-band GPRS transceiver module.

\section{LTCC SOP MODULE DESIGN}

The detailed block diagram for the antenna switch section of the proposed transceiver module is shown in Fig. 2. It consists of a diplexer separating the $900 \mathrm{MHz}$ EGSM frequency band from the $1800 / 1900 \mathrm{MHz}$ DCS/PCS frequency bands. Two sets of PIN diode based T/R switches for EGSM and DCS/PCS frequency bands are employed at the diplexer outputs. The $T / R$ switches are driven by the dc control voltage $V_{c t r 11}$ and $V_{c t r 2}$. For the input matching of GSM band SAW filter, a series inductor is included on the GSM receive path. For the DCS and PCS band receive paths, two transmission lines for diplexing the DCS and PCS receive bands are used. The characteristic impedance and the line length may be carefully designed to match the input impedances of DCS and PCS band SAW filters simultaneously.

By taking advantages of the 3-dimensional integration capability of LTCC substrate, most of the passive circuit components may be embedded in the substrate to minimize the module size. For the antenna switch section, the diplexer, two TX lowpass filters, two RF chokes, and the transmission lines are embedded in the LTCC substrate. The input matching circuits of SAW filters and LNA's, and the interconnections for the transceiver IC are also embedded. The multi-layered substrate has a dielectric constant $\varepsilon_{\mathrm{r}}$ of 9.4 , and a total substrate thickness of $0.8 \mathrm{~mm}$. The layout is carefully designed by invoking full-wave electromagnetic simulation to avoid possible interferences. The transceiver IC is mounted on the LTCC substrate in bare-die form, and the interconnections to the LTCC substrate are achieved by wire-bonding. Glob-top encapsulation is applied to protect the bare die. The PIN diodes, and two packaged SAW filters, one dual-SAW for EGSM/DCS band and one single-SAW for PCS band, along with some chip passive components are mounted by the SMT process. The photograph of the packaged module is shown in Fig. 3, and the module size is $13.8 * 8.1 * 1.8$ $\mathrm{mm}^{3}$.

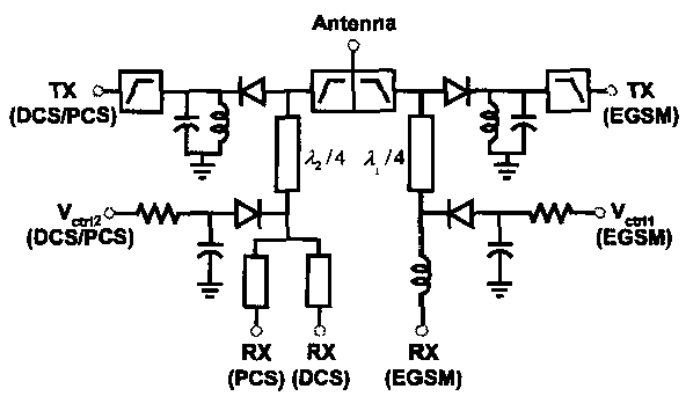

Fig. 2. Block diagram for the antenna switch section of the proposed tri-band GPRS transceiver module.

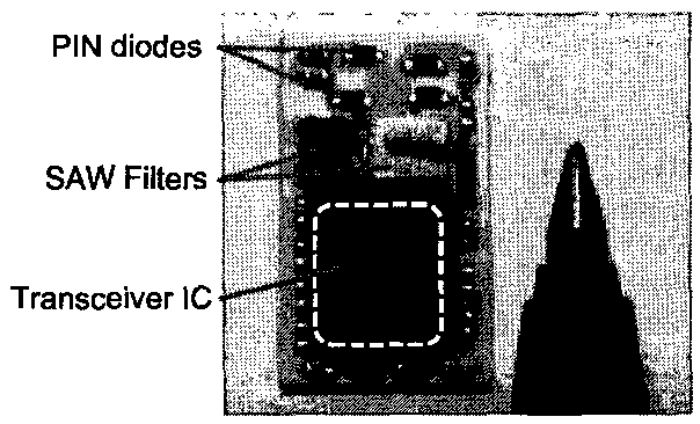

Fig. 3. Photograph of the proposed tri-band GPRS transceiver module.

\section{RESULTS}

Test sample kit for the antenna switch section of the proposed transceiver module is built to verify its performance separately. Shown in Fig. 4 is the measured 
frequency response for the EGSM-TX, from the TX port to the antenna port. The in-band (880 915 MHz) insertion loss is less than $1.1 \mathrm{~dB}$, and the return loss is greater than $18 \mathrm{~dB}$. The suppressions at $2^{\text {nd }}-, 3^{\text {rd }}-$, and $4^{\text {th }}$-harmonic frequencies are over $25 \mathrm{~dB}, 35 \mathrm{~dB}$, and $25 \mathrm{~dB}$, respectively. Frequency characteristics of the EGSM-RX portion, from antenna port to SAW filter output, are shown in Fig. 5. The in-band (925 960 MHz) insertion loss is less than $3.4 \mathrm{~dB}$, and the return loss is greater than $10.2 \mathrm{~dB}$.

The measured frequency responses of the DCS/PCS-TX, from TX port to antenna port, are shown in Fig. 6. The inband $(1710 \sim 1910 \mathrm{MHz})$ insertion loss is less than $1.3 \mathrm{~dB}$, and the return loss is greater than $17.8 \mathrm{~dB}$. The suppressions at $2^{\text {nd }}$ - and $3^{\text {rd }}$-harmonic frequencies are over $35 \mathrm{~dB}$ and $25 \mathrm{~dB}$, respectively. Frequency characteristics of the DCS-RX and PCS-RX, from the antenna port to SAW filter outputs, are shown in Fig. 7. The in-band $(1805 \sim 1880 \mathrm{MHz})$ insertion loss for DCS band is less than $3.62 \mathrm{~dB}$, and the return loss is greater than $10.8 \mathrm{~dB}$. The in-band $(1930 \sim 1990 \mathrm{MHz})$ insertion loss for PCS band is less than $3.73 \mathrm{~dB}$, with the return loss greater than $11.5 \mathrm{~dB}$.

The proposed LTCC transceiver module has been applied to a tri-band GPRS mobile phone design for integration test. The phone has passed the European telecommunications standards institute (ETSI) full-typeapproval (FTA) for GPRS mobile phone, and is currently available in the market. Typical measured receiver sensitivities for the EGSM, DCS, and PCS bands are $108.5 \mathrm{dBm},-106 \mathrm{dBm}$, and $-105.5 \mathrm{dBm}$, respectively. The transmit oscillators for EGSM and DCS/PCS bands have the typical output powers of $10 \mathrm{dBm}$, and $8 \mathrm{dBm}$, respectively. The rms phase error measured with an external power amplifier module is less than 2.8 degree for EGSM band and 3.6 degree for DCS/PCS band.

\section{CONCLUSION}

By taking advantage of the vertical integration capability of LTCC substrate, a very compact RF transceiver module for tri-band GPRS mobile application has been built. It integrates the tri-band antenna switch, SAW filters, and the transceiver IC into one single LTCC package. The proposed transceiver module complies the ETSI regulations, and its performance has been verified in a commercially available mobile phone. When implementing the proposed RF transceiver module in a GPRS mobile communication device, one may have the advantages of smaller circuit area, reduced component count, lower assembly cost, faster assembly time, higher reliability, more consistent circuit performance, and easier to tune and calibrate.

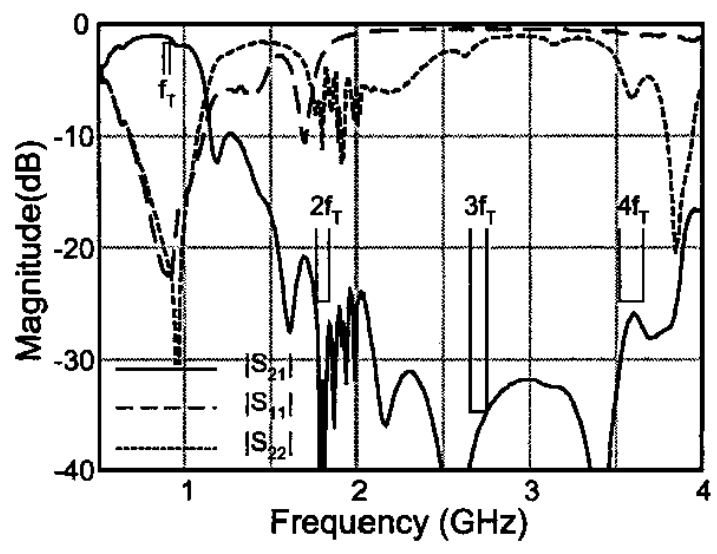

Fig. 4. Measured frequency responses of the EGSM-TX signal path for the antenna switch section of proposed tri-band GPRS transceiver module.

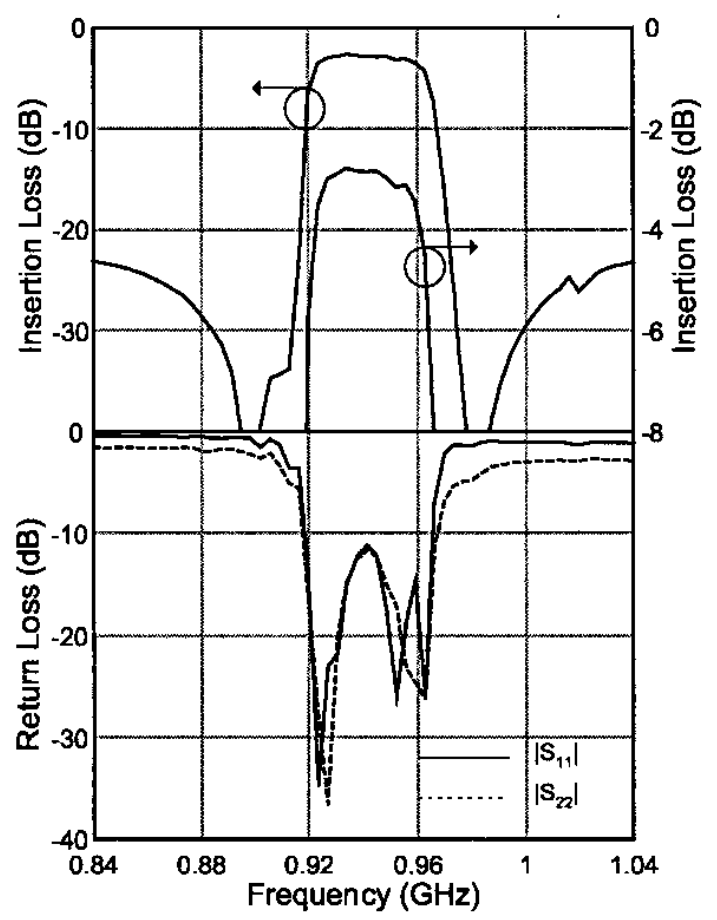

Fig. 5. Measured frequency responses of the EGSM-RX signal path for the antenna switch section of proposed tri-band GPRS transceiver module. 


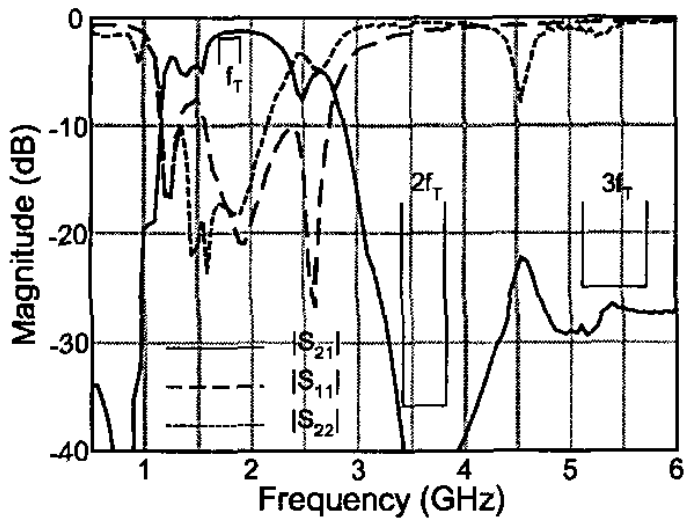

Fig. 6. Measured frequency responses of the DCS/PCS-TX signal path for the antenna switch section of proposed tri-band GPRS transceiver module.

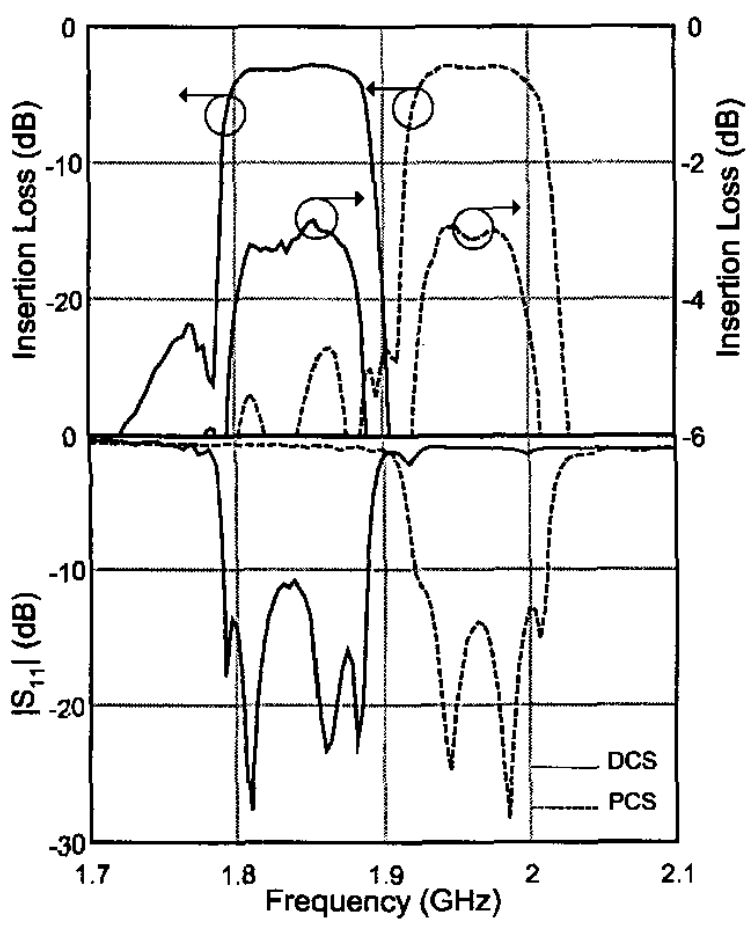

Fig. 7. Measured frequency responses of the DCS/PCS-RX signal paths for the antenna switch section of proposed triband GPRS transceiver module.

\section{REFERENCES}

[1] T. Watanabe, K. Furutani, N. Nakajima, and H. Mandai, "Antenna switch duplexer for dualband phone (GSM/DCS) using LTCC multiplayer Technology," in IEEE MTT-S Int. Microwave Symp. Dig., pp. 215-218, 1999.

[2] M. Hikita N. Matsuura, K. Yokoyama, N. Shibagaki, and K. Sakiyama, "SAW front-end module for GSM-based dual-band cellular phones with direct-conversion demodulation," IEEE Trans. Microwave Theory Tech., vol. 50 , pp. 2629-2638, Nov. 2002.

[3] R. Lucero, W. Qutteneh, A. Pavio, D. Meyers, and J. Estes, "Design of an LTCC switch diplexer front-end module for GSM/DCS/PCS applications," in IEEE Radio Frequency Integrated Circuit (RFIC) Symp. Dig., pp. 213-216, 2001.

[4] R. Lucero, A. Pavio, D. Penunuri, and J. Bost, "Design of an LTCC integrated tri-band direct conversion receiver front-end module," in IEEE MTT-S Int. Microwave Symp. Dig., pp. 1545-1548, 2002. 